be greater than in cold ones. An extreme case is that in which the temperature is so high as to produce general dissociation of the electrons from the nuclei, as appears to occur in the interior of stars. Apart from the disturbance produced by collisions, the magnetons are then free to set their axes parallel to the axis of rotation, and a large proportion of them would seem to do this in certain cases (vide infra). The upper limit of magnetisation is reached when all the magnetons are set parallel to the axis; this limit can be calculated in some cases, it being possible to estimate the number of electrons in electrostatically neutral matter of given kind and density. Other things being equal, the maximum magnetisation will be proportional to the density and independent of the speed of rotation.

The magnetic fields of the earth and sun can thus be accounted for on the present theory because these bodies are at a high temperature (in the case of the earth this is so except for the parts near the surface); what has hitherto been an obstacle in framing theories of their magnetic fields is here regarded as probably the determining factor as regards magnitude.

The same explanation applies to sun-spots. These occur in pairs of opposite magnetic polarity, and it has been pointed out that the polarities are such as would be accounted for by negative electricity in rotatory motion such as is observed (with opposite directions) in the members of a sun-spot pair. The sun-spot pair is thus regarded as the surface portion of a "line"-vortex continuously connecting the ends beneath the surface. The difficulty has been that no Stark effect is observable, such as would accompany rotating charges of the required amount; it has therefore been suggested that the magnetism of sun-spots is due to galvanic currents, i.e. electrons in translatorv motion through electrostatically neutral matter. This hypothesis is unnecessary; it seems possible to account for the observed fields, of order 3000 gauss, simply by the observed rotation of the matter composing the vortex, on the above magneton hypothesis.

The obliquity of the magnetic axes of the earth and sun requires some additional hypothesis for its explanation, since an unsymmetrical condition cannot be accounted for by a cause, like rotation, symmetrical about an axis. The obliquity being present, however, it seems possible to explain the secular variations of the solar and terrestrial magnetic fields as due to precessional motion of the magnetons. A magnetic field applied obliquely to a magneton will tend to cause it to precess round the lines of external magnetic force. The magnetic fields of the earth and sun, due mainly to rotation, will exert this effect on anv magnetons the axes of which are inclined to them; the speed of precession depends solely on the strength of the applied field if the magnetons are free, and the direction of precession agrees with the direction of rotation of the masnetic axes of the earth and sun.

The University, Manchester, November I3.

\section{Physiological Effects of Alcohol.}

DURING recent years a good deal of work has been done and a great many results have been published on the above subject. Some of these have been very recently reported in the general Press. In most, if not in all, of the researches which have been made on the physiological effects of moderate doses of alcohol, taken in the form of beer, spirits, or other alcoholic beverages, but little attention has been paid to the very important disturbing influence of what may be called "secondary products"- -whether extractive matters normal to the beverages in question or volatile by-products of the alcoholic fermentation process itself. Most people are aware that there is no definite relationship between the intoxicating efiects of certain wines and the amounts of alcohol which they contain, and the marked difference in the physiological effects of new whisky on one hand, and of well-matured spirit of the same alcoholic strength on the other, is a matter of common experience.

In the case of beer the soporific effect depends not a little on the hop and other extractives, and consequently there is no scientific justification for drawing any definite conclusions of a quantitative character as to the physiological effects of small doses of alcohol unless the beer used in the whole series of experiments had been of precisely the same character throughout, and even then the "personal equation" would introduce a further serious difficulty. Most beer-drinkers of middle age are well aware that the physiological effects of bitter beer on one hand, and of mild ale or lager (which are less heavily hopped) on the other, are not by any means the same, even for equal percentages of alcohol. The investigation of the effects of these various "secondary" constituents undoubtedly presents great experimental difficulties, but until their physiological significance is better understood there must always be an element of uncertainty in any conclusions arrived at in regard to the physiological effects of small doses of alcohol taken in the form of the usual alcoholic beverages.

London, November $\mathrm{r}_{5}$.

\section{A. Chaston Chapman.}

\section{Atomic Structure.}

THE arrangement of the non-nuclear electrons in the atom is being determined by work along two lines: first, from the Bohr-Sommerfeld theory of characteristic frequencies, and, secondly, from the Lewis-Langmuir or Born-Lande theory of the structure of molecules, supported by Prof. W. L. Bragg's work on atomic radii in crystals. It has been stated several times recently that the conclusions drawn from the two sources are mutually inconsistent, in that the first indicates that the electrons are revolving in planetary orbits, and the second that they are fixed in constant positions. The essential truth of the first theory is now beyond doubt; the second is extremely plausible. If they are really inconsistent the position would be intolerable.

But they are not really inconsistent. The BohrSommerfeld theory does not make explicit use of the assumption that the electrons in their stable states are moving; it assumes only that in those states they have the energy, calculated by Hamiltonian (relativity) dynamics, which they would have if they were moving in certain orbits. It is not logically impossible to maintain that they have that energy and are yet at rest. Nor is it physically impossible if we accept Bohr's principle of "correspondence," which has been so astoundingly successful in explaining the Stark effect and in predicting the number of components in lines of the hydrogen and helium spectra. According to that principle, the intensity and polarisation of components can be predicted by the application of classical dynamics to certain assumed orbits, although it must be assumed at the same time that the electrons are not moving in those orbits. If intensity and polarisation can be predicted from orbits that are wholly fictitious, why not energy?

Of course, the adoption of Bohr's principle in this extreme form would sever the last connection between classical dynamics and the real structure of the atom. But we are surely now all convinced that we must abandon part of that connection. Is there anv reason 